\title{
Influência do vigor de sementes na resposta do fejoeiro à adubação com fósforo
}

\author{
Influence of seed strength on bean response to phosphorus fertilization \\ Influencia del vigor de las semillas en respuestas del frijol a la fertilización con fósforo
}

Recebido: 19/01/2022 | Revisado: 27/01/2022 | Aceito: 04/02/2022 | Publicado: 06/02/2022

\author{
Pedro Ruben Viera Fariña \\ ORCID: https://orcid.org/0000-0003-1512-1246 \\ Universidade Federal de Viçosa, Brasil \\ E-mail: rubenviera20@gmail.com \\ Marcos Fabian Sanabria Franco \\ ORCID: https://orcid.org/0000-0002-7820-9037 \\ Universidade de São Paulo, Brasil \\ E-mail: marcosfabiansanabria@gmail.com \\ Leonardo Angelo Aquino \\ ORCID: https://orcid.org/0000-0002-7764-730X \\ Universidade Federal de Viçosa, Brasil \\ E-mail: aquino.ufv@gmail.com \\ Willian Rodrigues Macedo \\ ORCID: https://orcid.org/0000-0003-4660-913X \\ Universidade Federal de Viçosa, Brasil \\ E-mail: willian_rmacedo@yahoo.com.br \\ Volnei Pauletti \\ ORCID: https://orcid.org/0000-0002-9231-7851 \\ Universidade Federal do Paraná, Brasil \\ E-mail: vpauletti@ufpr.br \\ Carlos Diego da Silva \\ ORCID: https://orcid.org/0000-0003-4674-8455 \\ Universidade de São Paulo, Brasil \\ E-mail: carlosdiego@usp.br
}

\begin{abstract}
Resumo
Sementes de alto vigor resultam em melhor estabelecimento da planta e podem com isso melhorar o aproveitamento de nutrientes, especialmente do fósforo (P). Desta forma, objetivou-se avaliar o crescimento e absorção de P pelo feijoeiro em função do vigor das sementes em solos de texturas contratantes. Os tratamentos foram montados em esquema fatorial $2 \times 5$, combinando alto e baixo vigor de semente com cinco doses de $\mathrm{P}$ que foram $0,35,70,140$ e $280 \mathrm{mg} \mathrm{dm}^{-3} \mathrm{em} \mathrm{solo}$ de textura média e de $0,50,100,200,400 \mathrm{mg} \mathrm{dm}^{-3} \mathrm{em}$ solo argiloso. Foram cultivadas quatro plantas do cultivar Dama em vasos contendo $5 \mathrm{dm}^{3}$ de solo e avaliadas até início de floração. Sementes de alto vigor resultaram em incremento da matéria seca da planta (parte aérea + raiz) de 11 e $15 \%$ nos solos de textura media e argiloso, respectivamente. A fotossíntese aumentou nas plantas originadas de sementes de alto vigor em $26,04 \%$ no solo de textura media, mas foi similar entre as plantas de sementes de alto e baixo vigor no solo argiloso. A dose de P necessária para alcance de pelo menos $95 \%$ da máxima fotossíntese foi $46 \%$ maior nas plantas originadas de sementes de baixo vigor. $\mathrm{O}$ acúmulo de $\mathrm{P}$ das plantas de sementes de alto vigor cultivadas em solo de textura média foi $37 \%$ maior do que as de baixo vigor. No solo argiloso não houve esta diferença, no entanto, a dose de $\mathrm{P}$ no solo para o alcance do máximo acúmulo foi menor quando as sementes foram de alto vigor. A taxa de recuperação do $\mathrm{P}$ foi maior em plantas originadas de sementes de alto vigor nos dois solos. Conclui-se que as plantas oriundas de sementes de alto vigor apresentam maior crescimento e acúmulo do $\mathrm{P}$ o que evidência a importância da utilização de sementes de alto vigor para o maior aproveitamento das adubações fosfatadas no feijoeiro.
\end{abstract}

Palavras-chave: Phaseolus vulgaris; Taxa de recuperação; Capacidade tampão de P.

\begin{abstract}
Seeds with high vigor result in better plant establishment and can therefore improve the use of nutrients, especially phosphorus (P). Thus, the objective was to evaluate the growth and $\mathrm{P}$ absorption by the common bean as a function of seed vigor in soils with contracting textures. The treatments were set up in a $2 \times 5$ factorial scheme, combining high and low seed vigor with five doses of $\mathrm{P}$ that were $0,35,70,140$ and $280 \mathrm{mg} \mathrm{dm}-3$ in medium texture soil and $0,50,100$, $200,400 \mathrm{mg} \mathrm{dm}^{-3}$ in clayey soil. Four plants of the cultivar Dama were grown in pots containing $5 \mathrm{dm}^{-3}$ of soil and evaluated until flowering began. High vigor seeds resulted in an increase in plant dry matter (shoot + root) of 11 and $15 \%$ in medium textured and clayey soils, respectively. Photosynthesis increased in plants originating from high vigor seeds by $26.04 \%$ in medium textured soil, but it was similar between high and low vigor seed plants in clayey soil. The dose of P necessary to reach at least $95 \%$ of maximum photosynthesis was $46 \%$ higher in plants originated from seeds with low vigor. The accumulation of $\mathrm{P}$ in plants of high vigor seeds cultivated in medium-textured soil was $37 \%$ greater
\end{abstract}


than that of low vigor seeds. In clayey soil there was no such difference, however, the dose of P in the soil to reach the maximum accumulation was lower when the seeds were of high vigor. The $\mathrm{P}$ recovery rate was higher in plants originated from seeds with high vigor in both soils. It is concluded that plants from seeds with high vigor present greater growth and accumulation of $\mathrm{P}$, which highlights the importance of using seeds with high vigor for a better use of phosphate fertilizers in common bean.

Keywords: Phaseolus vulgaris; Recovery rate; Buffer capacity of P.

\begin{abstract}
Resumen
Las semillas con alto vigor dan como resultado un mejor establecimiento de la planta y, por lo tanto, pueden mejorar el uso de nutrientes, especialmente fósforo (P). Así, el objetivo fue evaluar el crecimiento y absorción de P por el frijol común en función del vigor de la semilla en suelos con texturas contraídas. Los tratamientos se configuraron en un esquema factorial $2 \times 5$, combinando alto y bajo vigor de semilla con cinco dosis de P que fueron $0,35,70,140$ y 280 $\mathrm{mg} \mathrm{dm}{ }^{-3}$ en suelo de textura media y $0,50,100,200,400 \mathrm{mg} \mathrm{dm}^{-3}$ en suelo arcilloso. Se cultivaron cuatro plantas del cultivar Dama en macetas que contenían $5 \mathrm{dm} 3$ de suelo y se evaluaron hasta que comenzó la floración. Las semillas de alto vigor dieron como resultado un aumento de la materia seca de la planta (brote + raíz) de 11 y $15 \%$ en suelos de textura media y arcillosos, respectivamente. La fotosíntesis aumentó en plantas originadas de semillas de alto vigor en un $26.04 \%$ en suelos de textura media, pero fue similar entre plantas de semillas de alto y bajo vigor en suelos arcillosos. La dosis de P necesaria para alcanzar al menos el $95 \%$ de la fotosíntesis máxima fue un $46 \%$ mayor en plantas originadas a partir de semillas de bajo vigor. La acumulación de $\mathrm{P}$ en plantas de semillas de alto vigor cultivadas en suelo de textura media fue $37 \%$ mayor que la de semillas de bajo vigor. En suelo arcilloso no hubo tal diferencia, sin embargo, la dosis de $\mathrm{P}$ en el suelo para alcanzar la acumulación máxima fue menor cuando las semillas fueron de alto vigor. La tasa de recuperación de $\mathrm{P}$ fue mayor en plantas provenientes de semillas de alto vigor en ambos suelos. Se concluye que las plantas provenientes de semillas con alto vigor presentan mayor crecimiento y acumulación de $\mathrm{P}$, lo que resalta la importancia de utilizar semillas con alto vigor para un mejor aprovechamiento de fertilizantes fosfatados en frijol común.
\end{abstract}

Palabras clave: Phaseolus vulgaris; Índice de recuperación; Capacidad tampón de P.

\title{
1. Introdução
}

O vigor das sementes é um dos principais atributos da qualidade fisiológica a ser considerado na implantação de uma lavoura. Sementes de alto vigor asseguram adequada população de plantas e constitui-se em uma ferramenta de extrema importância para alto rendimento nas lavouras (Mondo et al., 2016; Scheeren et al., 2010). Além disso, diferenças na velocidade de emergência proporcionam desuniformidade no crescimento inicial das plantas aumentando a competição intraespecífica (Pommel et al., 2002).

O feijão-comum no Brasil, é cultivado em mais de 3.990.000 ha em 10 estados e se constitui alimento básico do brasileiro (Borém et al., 2015). A produção de sementes de alta qualidade desta espécie depende especialmente do clima e da nutrição adequada da planta, fatores que conferem ganho de produtividade e a obtenção de sementes de alta qualidade (Farinelli et al., 2006).

Apesar da importância econômica e social da cultura do feijão é comum a utilização de sementes de baixa qualidade na produção desta leguminosa. O vigor da semente inclui todas as características que podem causar a emergência rápida e uniforme das plantulas em um amplo espectro de condicionamento ambiental após a semeadura. É uma característica complexa que depende da genética, condições ambientais do cultivo da planta, da colheita, benefeciamento e processo de armazenamento (Rezapour et al., 2013).

O feijoeiro comum é frequentemente cultivado em solos com baixa disponibilidade de fósforo (P) e esta é uma das principais limitações em sua produção aos cultivos em solos tropicais (Silva et al., 2014). O P é o nutriente que possui elevada interação com o solo e é o quarto nutriente mais absorvido pelas plantas de feijão. Para a produção de uma tonelada de grãos de feijão, considerando a planta inteira, a absorção é de 9 kg (NEPAR, 2019, Varennes et al., 2002). A disponibilidade de P para as plantas normalmente está associada a uma fração na solução e à outra presente na fase sólida do solo, também denominada de fração lábil, cujos valores de P se relacionam com as quantidades absorvidas pelas plantas. Os fatores de solo que influenciam na disponibilidade desse nutriente para as plantas são a umidade, o teor de argila e o poder tampão de P. Em solos argilosos, o valor do teor crítico de $\mathrm{P}$ para as culturas, determinado pelo método Mehlich-1, é menor do que em solos arenosos. A absorção 
de P pelas plantas só ocorre quando este está na forma iônica na solução do solo, em contato com a raiz, o que ocorre basicamente pela difusão, e, ao absorver o P da solução do solo em contato com a raiz, diminui o teor, formando um gradiente que propicia a difusão de um ponto de maior concentração para um de menor, em um processo dinâmico (Schlindwein \& Gianello, 2009, Graciano et al., 2006, CQFS-RS/SC, 2004)

A eficiência agronômica dos adubos fosfatados pode ser influenciada pelas fontes de P, propriedades do solo, modos de aplicação e espécies vegetais (Correa et al.,2004). A recuperação do P aplicado via fertilizantes, pode ser aumentada pelo maior crescimento do sistema radicular. Maior extensão de raízes aumenta a interceptação e facilita o fluxo difusivo de P por reduzir as distâncias para transporte de P no solo. Sementes vigorosas podem gerar plântulas com sistema radicular mais robusto e mais eficientes na absorção do P (Liu et al., 2015), o que pode reduzir a perda do nutriente para o processo de adsorção no solo. Assim, objetivou-se avaliar o crescimento vegetal à absorção de $\mathrm{P}$ pelo feijoeiro em função do vigor das sementes em solos de texturas contratantes.

\section{Material e Métodos}

Dois experimentos foram conduzidos em casa de vegetação utilizando um Latossolo Vermelho-Amarelo com textura argilosa e um Latossolo Amarelo de textura média, cujos atributos químicos e físicos constam na Tabela 1.

Tabela 1. Análise química e física do solo coletado antes da instalação do experimento.

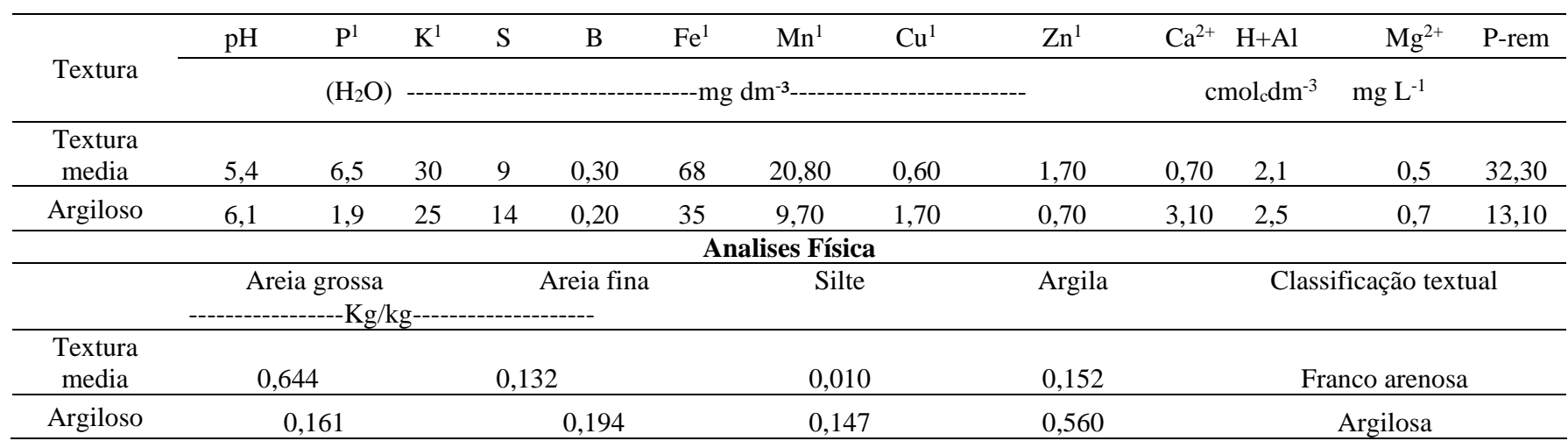

${ }^{1}$ Extrator de P: Mehlich-1; ${ }^{2}$ Fósforos remanescente. Fonte: Autores.

Em cada experimento os tratamentos foram arranjados em esquema fatorial 2 x 5 compostos da combinação de sementes com alto e baixo vigor com cinco doses de P: 0,35, 70, 140, $280 \mathrm{mg} \mathrm{dm}^{-3}$ no solo de textura media e 0,50, 100, 200 e 400 mg $\mathrm{dm}^{-3}$ no argiloso. A fonte de $\mathrm{P}$ foi o superfosfato triplo com $46 \%$ de $\mathrm{P}_{2} \mathrm{O}_{5}$. O delineamento experimental utilizado foi o inteiramente casualizado com cinco repetições. A unidade experimental foi composta por vasos preenchidos com $5 \mathrm{dm}{ }^{-3} \mathrm{de}$ solo com quatro plantas de feijoeiro. A cultivar utilizado foi a Dama, do grupo carioca, com ciclo 85-95 dias, habito indeterminado tipo III semi-ereto a prostrado.

Os vasos foram preenchidos com solo corrigido com calcário e adubado com 210, 200, 100, 3,75 e 1,92 $\mathrm{mg} \mathrm{dm}^{-3}$ de $\left(\mathrm{NH}_{4}\right)_{2} \mathrm{SO}_{4}, \mathrm{KNO}_{3}, \mathrm{NH}_{4} \mathrm{NO}_{3}, \mathrm{ZnSO}_{4} \mathrm{e} \mathrm{CuSO}_{4}$, respectivamente. Foram semeadas 10 sementes por vaso e após a emergência foi efetuado o desbaste para remanescerem quatro plantas por vaso. As doses de P foram aplicadas por ocasião da semeadura e o adubo misturado em todo o volume de solo do vaso.

Os lotes de sementes foram caracterizados quanto ao potencial fisiológico (RAS, 2009). Para a obtenção de sementes de baixo vigor foi feito envelhecimento acelerado pelo método gerbox (Filho, 1999). Posteriormente foi feita teste de germinação a partir de sementes envelhecidas e não envelhecidas que apresentaram 72 e $99 \%$ de germinação nos 12 dias, respetivamente. O 
lote de sementes de alto vigor apresentou emergência em campo de 80, 95, 99\% e o lote de baixo vigor de 40, 55, 60\% aos 6, 10, 12 dias após da semeadura, respectivamente.

Aos 40 dias após a semeadura, entre 8:00 e 10:00 horas foi determinada a taxa fotossintética $\left(\mu \mathrm{mol} \mathrm{CO}_{2} \mathrm{~m}^{-2} \mathrm{~s}^{-1}\right)$. Para isso foi quantificado o fluxo de fótons $\left(1.000 \mathrm{mmol} \mathrm{m}^{-2} \mathrm{~s}^{-1}\right)$ e concentração ambiente de $\mathrm{CO}_{2}$, com o uso de analisador de gás infravermelho portátil, modelo LICOR 6400XT (Li-COR, Lincoln, NE, EUA).

No estádio R5 as plantas foram colhidas e separadas em parte aérea e raízes. Essas partes foram secas em estufa com circulação forçada de ar a $70{ }^{\circ} \mathrm{C}$, por 72 horas. Posteriormente a parte aérea e raízes secas foram quantificadas em massa e trituradas em moinho tipo Willey equipado com peneira de 1,27 mm. As amostras foram submetidas à digestão sulfúrica e quantificados os nutrientes N e P (EMBRAPA 2009).

Amostras de solo foram coletadas por ocasião da colheita das plantas para determinação da disponibilidade de P pelo extrator Mehlich-1 (Donagema et al., 2011).

A taxa de recuperação do $\mathrm{P}$ aplicado via fertilizante (TR) foi calculada com a seguinte fórmula: TR $(\%)=(\mathrm{P}$ acumulado nas plantas adubadas - P acumulado nas plantas sem adubação com P) / Dose de P aplicada × 100 (Maluf et al., 2015).

Inicialmente os dados foram submetidos à verificação da normalidade e homogeneidade das variâncias. Posteriormente os dados foram submetidos à análise de variância (ANOVA) e regressão. As médias do vigor de sementes foram comparadas pelo teste F. Para as análises estatísticas foi utilizado o Software em planilha eletrônica SPEED Stat (Carvalho e Mendes, 2017) e adotada a significância de $5 \%$.

\section{Resultados}

Houve incremento da matéria seca total das plantas em função das doses de P em ambos os solos (Figura 1a e 1b). No solo de textura media as plantas oriundas das sementes de alto vigor atingiram $24,03 \mathrm{~g} \mathrm{vaso}^{-1}$ de matéria seca com a maior dose de P aplicada. Quando as plantas foram oriundas das sementes de baixo vigor a máxima matéria seca foi de $21,2 \mathrm{~g}$ vaso ${ }^{-1} \mathrm{obtida}$ com a dose de $184 \mathrm{mg} \mathrm{dm}^{-3}$ de P. No cultivo em solo argiloso a matéria seca total de plantas foi incrementada até a maior dose para as plantas originadas tanto das sementes de baixo como alto vigor. As sementes de alto vigor resultaram em $24,76 \mathrm{~g}$ vaso ${ }^{-1}$ de matéria seca total, enquanto que as oriundas das sementes de baixo vigor produziram apenas $21,0 \mathrm{~g}$ vaso ${ }^{-1}$. Para obtenção de 95\% das máximas produções de matéria seca foram necessários 190 e $297 \mathrm{mg} \mathrm{dm}^{-3}$ de P no solo quando as sementes utilizadas foram de alto e baixo vigor, respectivamente. 
Figura 1. Matéria seca (parte aérea e raiz) em função do vigor da semente do feijoeiro e de doses de $\mathrm{P}$ em solos de textura media (a) e argiloso (b). UFV, Rio Paranaíba - MG (2020).

(a) $\mathrm{F}_{\text {vigor: }} 93.12^{* *} \mathrm{~F}_{\text {doses: } 119.29^{* *}} \mathrm{~F}_{\text {interação:9,43** }} \quad$ (b) $\mathrm{F}_{\text {vigor: } 233.65^{* *}} \mathrm{~F}_{\text {doses: }}$ 447,45** $\mathrm{F}_{\text {interação:9.6** }}$

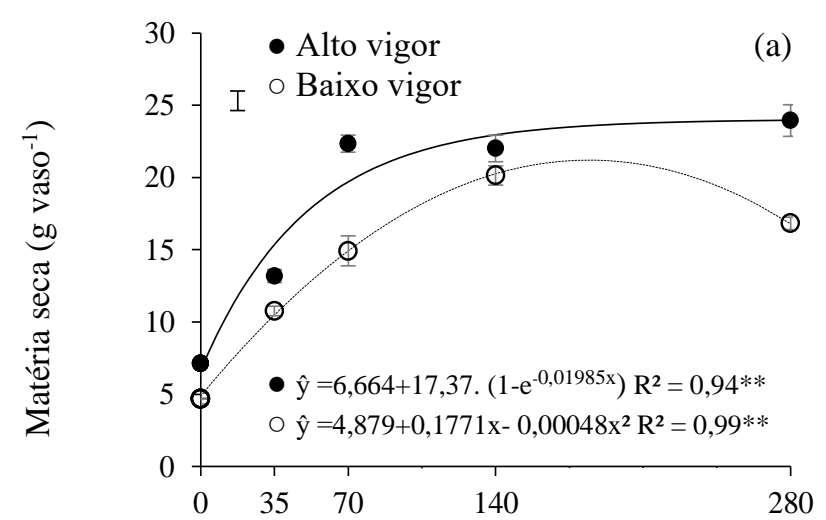

Doses de $\mathrm{P}\left(\mathrm{mg} \mathrm{dm}^{-3}\right)$

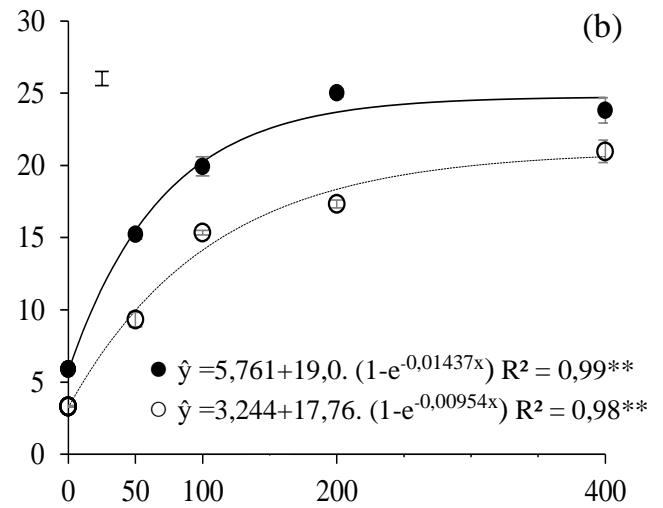

Doses de $\mathrm{P}\left(\mathrm{mg} \mathrm{dm}^{-3}\right)$

Fonte: Autores.

$\mathrm{O}$ aumento das doses de $\mathrm{P}$ resultou em incremento da taxa assimilatória líquida de $\mathrm{CO}_{2}$ nas plantas cultivadas em ambos os solos (Figura 2a e 2b). As plantas cultivadas em solo de textura media, apresentaram maior incremento de fotossíntese com a aplicação de $\mathrm{P}$ quando as sementes utilizadas foram de alto vigor. No solo argiloso as maiores fotossínteses foram semelhantes nas plantas oriundas de sementes de alto ou baixo vigor, sendo necessários 119,2 e 174,1 mg dm³ de P para alcançar pelo menos 95\% da maior fotossíntese, respectivamente

Figura 2. Taxa assimilatória líquida de $\mathrm{CO}_{2}(A)$ em função do vigor da semente do feijoeiro e de doses de $\mathrm{P}$ em solo de textura media (a) e argiloso (b). UFV, Rio Paranaíba - MG (2020).

$\mathrm{F}_{\text {vigor: } 63,91 * *} \mathrm{~F}_{\text {doses: } 28,38^{* *}} \mathrm{~F}_{\text {interação:1,19 ns }} \quad$ (b) $\mathrm{F}_{\text {vigor: } 2,43 \mathrm{~ns}} \mathrm{~F}_{\text {doses: } 364,99 * *} \mathrm{~F}_{\text {interação: } 9,46 * *}$

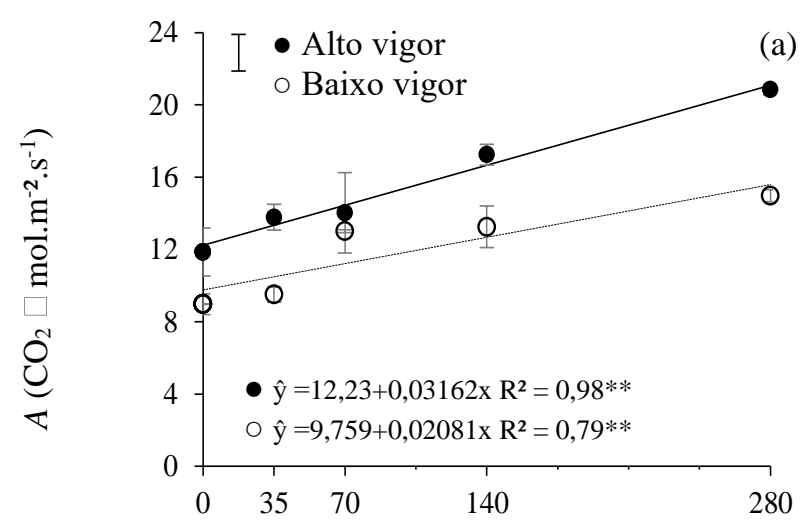

Doses de $\mathrm{P}\left(\mathrm{mg} \mathrm{dm}^{-3}\right)$

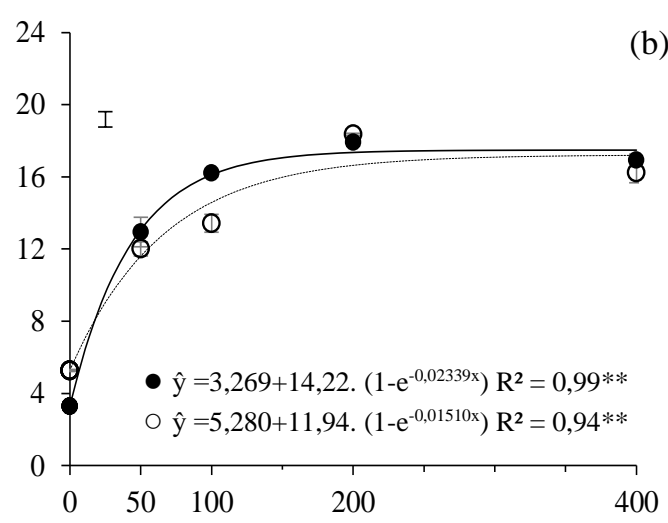

Doses de $\mathrm{P}\left(\mathrm{mg} \mathrm{dm}^{-3}\right)$

Fonte: Autores.

Houve incremento do acúmulo de $\mathrm{P}$ até a maior dose de $\mathrm{P}$ para ambos os solos (Figura 3a e 3b). No solo de textura media o maior acúmulo de $\mathrm{P}$ foi de 152,8 mg vaso quando utilizadas sementes de alto vigor e de 95,64 mg vaso quando utilizadas as sementes de baixo vigor. No solo argiloso os acúmulos de P foram de 31,62 e de 30,32 mg vaso com as sementes de alto e 
baixo vigor, respectivamente. Para o alcance de $95 \%$ do maior acúmulo de $\mathrm{P}$ das plantas cultivadas no solo argiloso foi necessário 98,9 e 158,4 $\mathrm{mg} \mathrm{dm}^{-3}$ de $\mathrm{P}$ quando utilizadas sementes de alto e baixo vigor, respectivamente.

Figura 3. Fósforo (P) acumulado (parte aérea + raízes) em função do vigor da semente do feijoeiro e de doses de $\mathrm{P}$ em solo de textura media (a) e argiloso (b). UFV, Rio Paranaíba - MG (2020).

$\mathrm{F}_{\text {vigor: } 96,61 * *} \mathrm{~F}_{\text {doses: } 328,13^{* *}} \mathrm{~F}_{\text {interação:36,69 ns }} \quad$ (b) $\mathrm{F}_{\text {vigor: } 31,40 * *} \mathrm{~F}_{\text {doses: } 258,27 * *} \mathrm{~F}_{\text {interação: } 4,59 * *}$

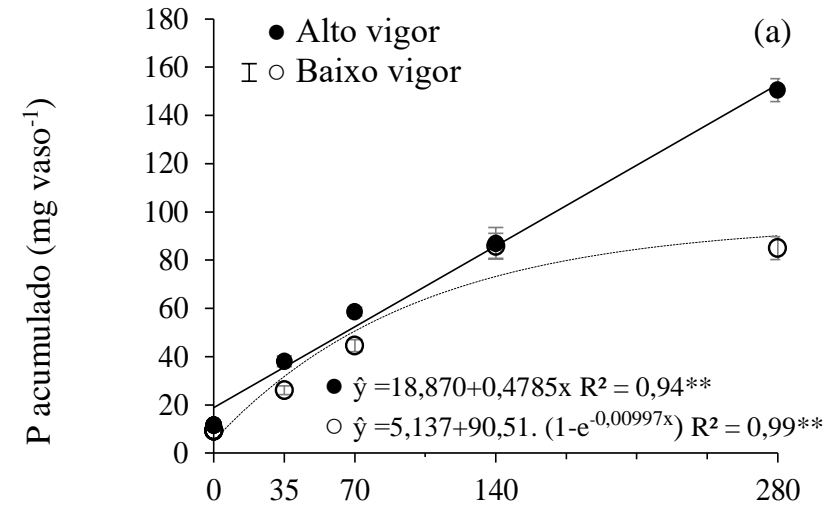

Doses de $\mathrm{P}\left(\mathrm{mg} \mathrm{dm}^{-3}\right)$

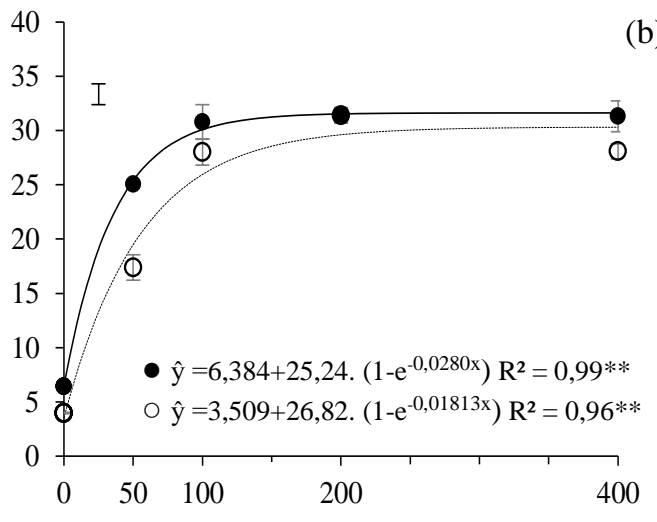

Doses de $\mathrm{P}\left(\mathrm{mg} \mathrm{dm}^{-3}\right)$

Fonte: Autores.

$\mathrm{O}$ acúmulo de $\mathrm{N}$ nas folhas foi incrementado até a maior dose de $\mathrm{P}$ aplicada nas plantas cultivadas no solo de textura media com maiores acúmulos de 95,64 e 91,02 $\mathrm{mg} \mathrm{dm}^{-3}$ quando utilizadas sementes de alto e baixo vigor, respectivamente (Figura 4a). Para obtenção de 95\% dos máximos acúmulos foliares de $\mathrm{N}$ foi necessário 115,1 e 151,6 mg dm³ de P quando as sementes utilizadas foram de alto e de baixo vigor, respectivamente. No solo argiloso o máximo acúmulo foliar de $\mathrm{N}$ de $97,4 \mathrm{mg}$ vaso foi obtido nas plantas oriundas de sementes de alto vigor e adubadas com 246 mg dm³ de P (Figura 4b). Quando utilizadas sementes de baixo vigor o acúmulo foliar de $\mathrm{N}$ alcançou 86,31 mg vaso com a aplicação da maior dose de $\mathrm{P}$.

Figura 4. Nitrogênio (N) acumulado nas folhas em função do vigor da semente do feijoeiro e de doses de $\mathrm{P}$ em solo de textura media (a) e argiloso (b). UFV, Rio Paranaíba - MG (2020).

$\mathrm{F}_{\text {vigor: } 15,75^{* *}} \mathrm{~F}_{\text {doses: } 68,91 * *} \mathrm{~F}_{\text {interação:1,56 }}$

(b) $\mathrm{F}_{\text {vigor: } 84,22 * *} \mathrm{~F}_{\text {doses: }}$ 319,23** $\mathrm{F}_{\text {interação: } 7,39 * *}$

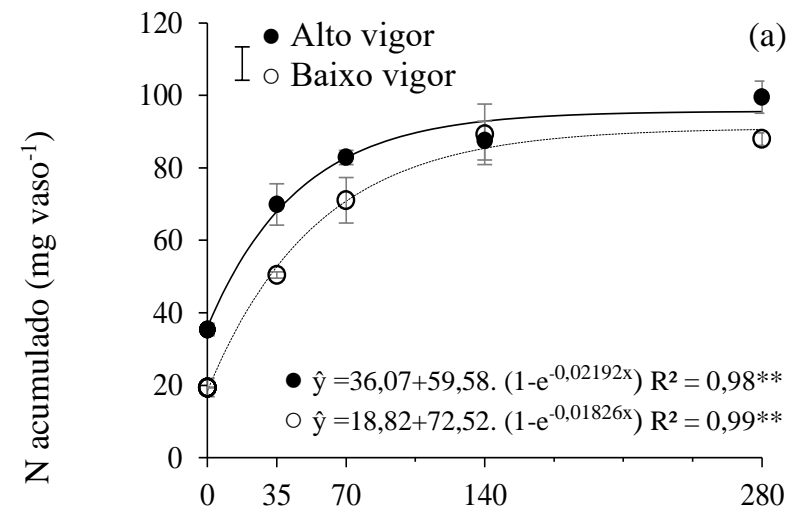

Doses de $\mathrm{P}\left(\mathrm{mg} \mathrm{dm}^{-3}\right)$

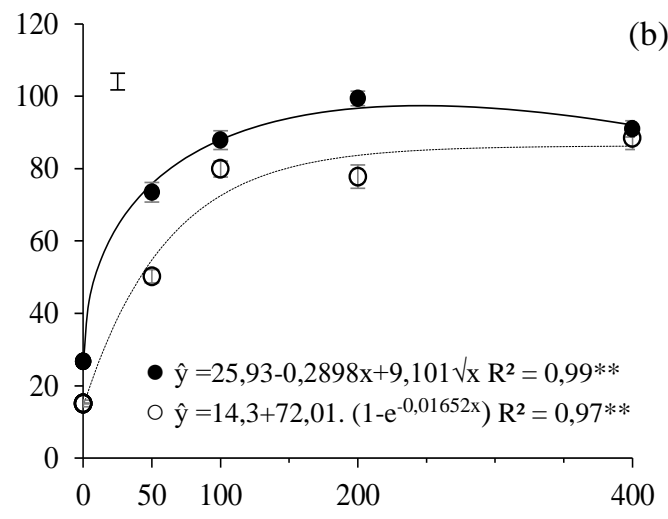

Doses de $\mathrm{P}\left(\mathrm{mg} \mathrm{dm}^{-3}\right)$

Fonte: Autores. 
Houve incremento da disponibilidade de $\mathrm{P}$ nos solos de textura media e argiloso em função das doses de $\mathrm{P}$ aplicadas (Figuras 5a e 5b). No solo de textura media após o cultivo do feijoeiro oriundo de sementes de baixo vigor a disponibilidade de P foi maior que após o cultivo com sementes de alto vigor. No solo argiloso o cultivo de plantas originadas de sementes de alto ou baixo vigor não alterou de a disponibilidade de $\mathrm{P}$ no solo. A disponibilidade de $\mathrm{P}$ no solo argiloso alcançou em média 24,4 $\mathrm{mg} \mathrm{dm}^{-3}$ de $\mathrm{P}$ com a maior dose aplicada do nutriente. No solo de textura media, essa disponibilidade foi incrementada para 28,1 e 40,8 $\mathrm{mg} \mathrm{dm}^{-3}$ com a maior dose de $\mathrm{P}$ quando cultivadas plantas oriundas de sementes de alto e baixo vigor, respectivamente.

Figura 5. P-disponível no solo extraído por Mehlich-1 após cultivo do feijoeiro em função do vigor da semente do feijoeiro e de doses de P em solo de textura media (a) e argiloso (b). UFV, Rio Paranaíba - MG (2020).
$\mathrm{F}_{\text {vigor: } 306,87 * *} \mathrm{~F}_{\text {doses: }}$ 702,59** $\mathrm{F}_{\text {interação:43,74** }}$
(b) $\mathrm{F}_{\text {vigor: } 119,33 * *} \mathrm{~F}_{\text {doses: }}$ 4337,84** $\mathrm{F}_{\text {interação: } 327,27 * *}$
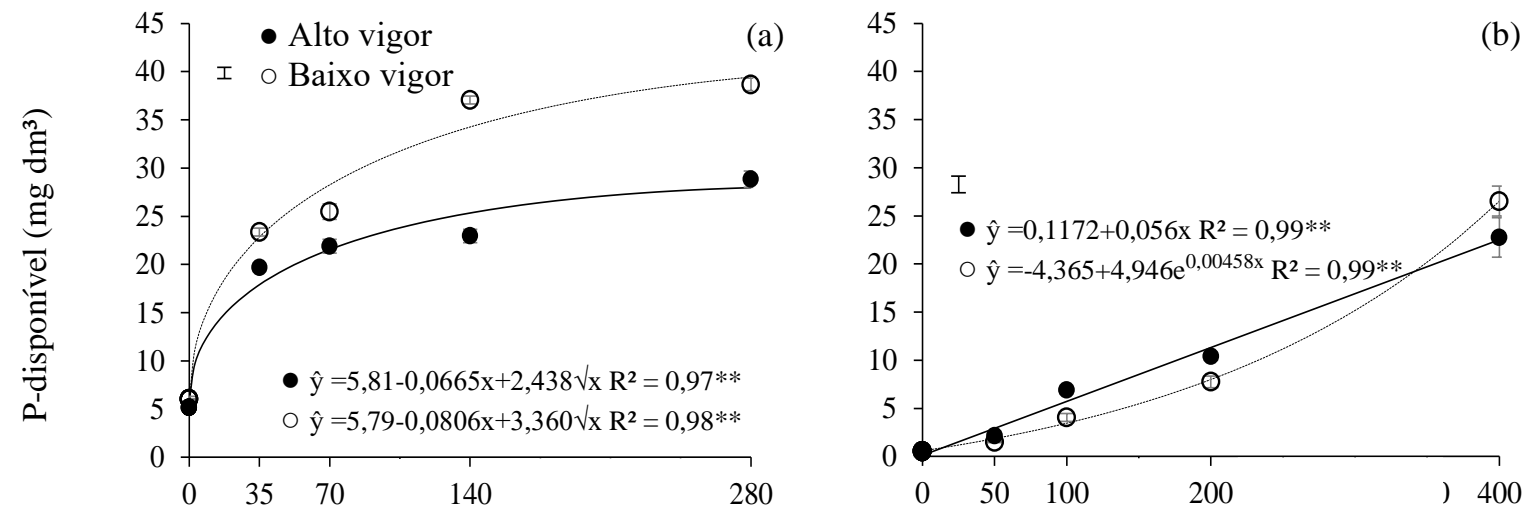

Doses de $\mathrm{P}\left(\mathrm{mg} \mathrm{dm}^{-3}\right)$

Doses de $\mathrm{P}\left(\mathrm{mg} \mathrm{dm}^{-3}\right)$

Fonte: Autores.

No cultivo em solo de textura media houve redução da taxa de recuperação de $\mathrm{P}$ em função das doses aplicadas no solo deste nutriente quando as sementes utilizadas foram de alto vigor. Quando as sementes foram as de baixo vigor a máxima taxa de recuperação de $10,8 \%$ foi obtida com a dose de $119 \mathrm{mg} \mathrm{dm}^{-3} \mathrm{de} \mathrm{P}$ (Figura 6a). No cultivo em solo argiloso, independentemente do vigor da semente houve redução da taxa de recuperação de P pelo feijoeiro em função das doses de $\mathrm{P}$ aplicadas (Figura 6b). Com a menor dose de $\mathrm{P}$ a recuperação foi maior quando utilizadas sementes de alto vigor. 
Figura 6. Taxa de recuperação de $\mathrm{P}$ em função do vigor da semente do feijoeiro e de doses de $\mathrm{P}$ em solo de textura media (a) e argiloso (b). UFV, Rio Paranaíba - MG (2020).

$\begin{array}{ll}\mathrm{F}_{\text {vigor: } 21,27 * *} \mathrm{~F}_{\text {doses: } 9,62 * *} \mathrm{~F}_{\text {interação: 3,06** }} & \text { (b) } \mathrm{F}_{\text {vigor: } 785,1 * *} \mathrm{~F}_{\text {doses: } 880,04 * *} \mathrm{~F}_{\text {interação: } 750,88 * *}\end{array}$

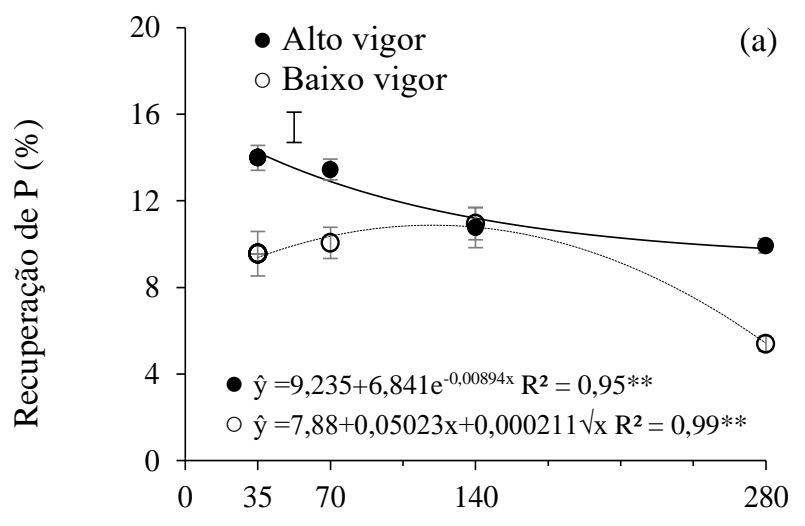

Doses de P $\left(\mathrm{mg} / \mathrm{dm}^{3}\right)$

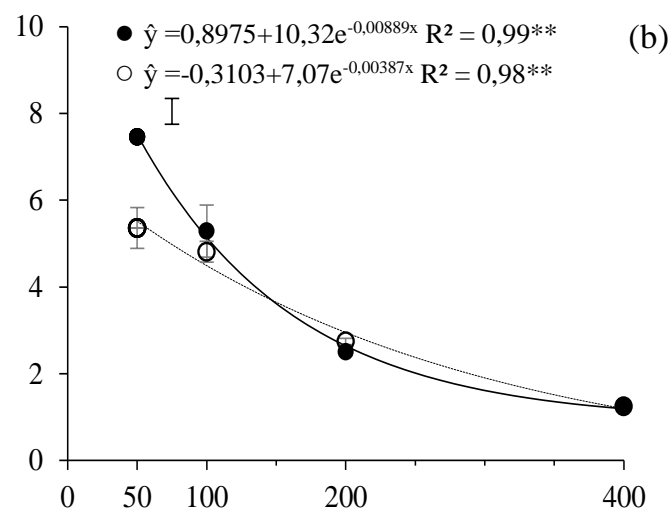

Doses de $\mathrm{P}\left(\mathrm{mg} / \mathrm{dm}^{3}\right)$

Fonte: Autores.

\section{Discussão}

A matéria seca das plantas (parte aérea + raízes) foi menor quando utilizadas sementes de baixo vigor em 11,8 e 15,2\% nos solos de textura media e argiloso, respectivamente. De forma similar, Zucareli et al. (2011) observaram que plantas de feijoeiro oriundas de sementes de alto vigor produziram aos 14 dias após a emergência, 6\% mais matéria seca que as plantas oriundas das sementes de baixo vigor (Plantas de soja advindas de sementes de alto vigor também apresentaram 14\% de aumento da matéria seca aos 28 dias em relação às de baixo vigor (Kolchinski et al., 2006). Semente de alto vigor pode originar plantas com sistema radicular mais expandido, o que é importante para o aproveitamento e exploração dos nutrientes aplicados no solo (Silva et al., 2014). O baixo vigor de sementes pode reduzir a velocidade de emergência e o crescimento inicial da planta (Henming et al., 2010).

O fósforo é responsável por $0,1 \%$ da matéria seca total das plantas, que varia de $0,2 \%$ a $0,4 \%$ nos tecidos foliares. Está presente em grande parte na forma inorgânica como componentes de ésteres de fosfato (fosfato de açúcar, nucleotídeos e fosfolipídios), certas enzimas e proteínas, adenosina tri-fosfato e está envolvido em várias reações de transferência de energia e informações genéticas (Bhattacharya, 2019). O fósforo é importante na construção de energia para o metabolismo do crescimento das plantas através de produções celulares, como ATP e ADP, desde os estágios iniciais até o final da vida útil da planta (Muaid 2014).

A taxa fotossintética aumentou com sementes de alto vigor no solo de textura media. No solo argiloso houve discreto aumento da taxa fotossintética nas plantas originadas das sementes de baixo vigor com maior dose de P (Figura 2). De forma similar na cultura de soja a taxa fotossintética aumentou com sementes de alto vigor, incrementando a matéria seca da folha (Pedó et al., 2015). A capacidade da planta de manter altas taxas de fotossíntese é importante para o aproveitamento das condições ideais de água no solo e temperatura para o crescimento da planta (Fenta et al., 2011). A baixa qualidade da semente pode reduzir a fotossíntese e de forma dependente da variedade (Silva et al., 2016). As plantas de sementes de alto vigor exploram maior volume de solo e mantém condições de nutrientes nos tecidos em condições ótimas por maior período de tempo, o que reflete no aumento da taxa fotossintética (Neves et al. 2009).

Houve maior acúmulo de $\mathrm{P}$ na planta de lote de alto vigor de $37 \%$ e 0,4 \% nos solos de textura media e argiloso, respetivamente (Figura 3). Resultado similar à pesquisa que evidenciou aumento de $66 \%$ no acúmulo de $\mathrm{P}$ na cultura de feijão 
provenientes de sementes de alto vigor, e maiores doses do nutriente aplicado no solo argiloso (Silva et al., 2002). A absorção de $\mathrm{P}$ gera zonas de depleção em torno a raízes existente, mas devido à baixa mobilidade no solo a aquisição de $\mathrm{P}$ é altamente dependente do crescimento contínuo da raiz e da exploração de novos domínios do solo que ainda não foram esgotados de $\mathrm{P}$ (Shanka et al., 2018). Algumas características fisiológicas da planta como a absorção do P e translocação desse nutriente estão controladas por genes com diferentes expressões em função da variedade (Silva et al., 2019). As plantas absorvem a maior parte do $\mathrm{P}$ na solução do solo, principalmente na forma de íons ortofosfatos $\left(\mathrm{H}_{2} \mathrm{PO}_{4} \mathrm{e} \mathrm{HPO}_{4}{ }^{2-)}\right.$, mediante a solubilização de minerais fosfatados e da mineralização da matéria orgânica (Dechen \& Nachtigall, 2007). O contato do íon-raiz ocorre por difusão, a pesar de baixa mobilidade no solo, na planta a mobilidade e alta com distribuição entre os órgãos, ou seja, das folhas velhas paras as novas e para os frutos e sementes (Silva, 2006).

$\mathrm{O}$ acúmulo de $\mathrm{N}$ foi 11 e $0,4 \%$ maior em sementes de alto vigor em solos de textura media e argiloso, respetivamente (Figura 4). O nitrogênio tem influencias no crescimento e morfologias radiculares que são determinantes da produtividade das plantas e sua disponibilidade e concentração interna influenciam na partição da biomassa entre raízes e folhas (Razaq et al., 2017). O P além de aportar energia para o melhor aproveitamento do N, também influencia no estabelecimento de nódulos ao aumentar de número de pelos radiculares e consequentemente mais sítios de infecção para bactérias fixadoras de $\mathrm{N}_{2}$ (Silva et al. 2010).

A disponibilidade de P no solo foi $31 \%$ maior no solo de textura media e 18\% maior no solo argiloso após o cultivo plantas oriundas de sementes de baixo vigor (Figura 5). Em solos de textura media, há maior disponibilidade de P uma vez que a adsorção deste nutriente é menor em função da baixa concentração de argila (Machado et al., 2011). Além disso ocorre precipitação deste elemento em solução com formas iônicas de $\mathrm{Fe}^{3+}, \mathrm{Al}^{3+} \mathrm{e} \mathrm{Ca}^{2+}$, e de maneira mais significativa sua adsorção pelos hidróxidos de $\mathrm{Fe}^{3+}$ e de $\mathrm{Al}^{3+}$ presentes em maiores quantidades nos solos mais intemperizados (Novais et al., 2007). Sementes de alto vigor proporcionam maior crescimento de sistema radicular, aumentando o $\mathrm{P}$ absorvido (Figura 3). Além disso plantas advindas de sementes vigorosas protegem o $\mathrm{P}$ da fase mineral do solo, com maior capacidade de fixação do $\mathrm{P}$ em formas não-lábeis (Correa et al. 2004).

Em solo de textura media, a recuperação de $\mathrm{P}$ foi de 10,8\% quando realizada a semeadura de sementes de alto vigor em relação às de baixo vigor. No solo argiloso independente do vigor houve redução da taxa de recuperação em função a doses de P aplicadas e essa taxa foi menor que a do solo de textura media (Figura 6). A taxa de recuperação é negativamente influenciada pela dose aplicada do nutriente (Mei et al., 2012) e comumente os solos de textura media propiciam maior recuperação do P que os solos argilosos (Bonfim et al. 2003, Ramos et al. 2010). A menor taxa de recuperação no solo argiloso pode ser atribuída ao aumento do poder tampão de fosfato desse solo (Bonfim et al.,2003). A taxa de recuperação correlacionou-se inversamente com a capacidade-tampão de $\mathrm{P}$ dos solos, assim, para a mesma quantidade de $\mathrm{P}$ absorvida, é necessária maior quantidade de $\mathrm{P}$ em solo com maior capacidade-tampão, como é o solo argiloso (Syers et al., 2008). Os solos de regiões tropicais, geralmente ácidos e com predominância de argilas sesquioxídicas, atuam como dreno do $\mathrm{P}$ aplicado e reduzem drasticamente o aproveitamento pelas plantas. Isso ocorre porque a absorção do $\mathrm{P}$ da solução do solo pelas plantas, que está em equilíbrio com a forma lábil, é negativamente relacionada com a energia de ligação de $\mathrm{P}$ e com o fator capacidade desse elemento no solo. Desse modo, em solo com maior fator capacidade há menor taxa de recuperação do P aplicado (Novais \& Smyth 1999).

\section{Conclusão}

Plantas oriundas de sementes de alto vigor produzem maior matéria seca, acumulam mais P e necessitam de menor dose de fertilizante fosfatado em relação às oriundas de sementes de baixo vigor. 


\section{Agradecimentos}

Ao CNPq - Conselho Nacional de Desenvolvimento Científico e Tecnológico, CAPES - Coordenação de Aperfeiçoamento de Pessoal de Nível Superior e FAPEMIG - Fundação de Amparo à Pesquisa do Estado de Minas Gerais pelo apoio financeiro à pesquisa.

\section{Referências}

Andrade C. A., Scapim, C. A., Lucca, A., \& Braccini, Martorelli, D. (2009). Produtividade, crescimento e partição de matéria seca em duas cultivares de feijão Acta Scientiarum Agronomy. 31(4), 683-688.

Anghinoni, I. (2007). Fertilidade do solo e seu manejo em plantio direto. In Novais, R.F., Alvarez V., V. H., Barros, N. F., Fontes, R. L. F., Cantarutti, R. B., Neves, J. C. L., Fertilidade do Solo. Viçosa, MG: Sociedade Brasileira de Ciência do Solo. 1017p.

Bertolin, D. C., Sá, M. E. D., \& Moreira, E. R. (2011). Parâmetros do teste de envelhecimento acelerado para determinação do vigor de sementes de feijão. Revista Brasileira de Sementes, 104-112.

Bhattacharya, A. (2019). Changing Environmental Condition and Phosphorus-Use Efficiency in Plants. Changing Climaté and Resource Use Efficiency in Plants, 241-305.

Bonfim-silva, E. M., Guimarães, S. L., Faria, L. N., Oliveira, J. R., Bosa, C. K., \& Fontenelli, J. V. (2014). Phosphorus fertilization on the development and production of pigeonpea in the cerrado oxisol in first cultivation. Bioscience Journal, 30, 1380-1388.

Borem, A., Carneiro, J. E. S., A cultura. In: Carneiro, J. E. S., Paula Júnior, T. J., \& Borem, A (Ed.). (2015) Feijão do plantio a colheita. Viçosa, MG: Universidade Federal de Viçosa. 1, 9 - 15.

Carvalho, A.M. X, \& Mendes, F.Q. (2017). Spreadsheet Program para Estatística Experimental e Descritiva. UFV.

Chuyong, G. B., Newbery, D. M., \& Songwe, N. C. (2000). Litter nutrients and retranslocation in a central African rain forest dominated by ectomycorrhizal trees. New Phytologist, 148(3), 493-510.

Comissão de Química e Fertilidade do Solo, CQFS-RS/SC. (2004). "Manual de adubação e calagem para os estados do Rio Grande do Sul e Santa Catarina." 401p-401p.

Corrêa, J. C., Mauad, M., \& Rosolem, C. A. (2004). Fósforo no solo e desenvolvimento de soja influenciados pela adubação fosfatada e cobertura vegetal. Pesquisa Agropecuária Brasileira, 39, 1231-1237.

Corte, V. B., Borges, E. E. L., Pontes C. A., Leite, I. T. A., Ventrella M. C, \& Mathias, A. A. (2006). Mobilização de reservas durante a germinação das sementes e crescimento das plântulas de Caesalpinia peltophoroides Benth. (leguminosae-caesalpinoideae). Revista Árvore, $30,941-949$.

Coutinho, P. W. R., Silva, D. M. S., Medeiros E. C., Okumura S. R. S., \& Júnior, M. L. S. (2014). Raté s of phosphorus fertilizers in the culture of cowpea in the northeastern on Staté of Pará. Revista Agro ambiente, 8, 66-73.

da Silva Bonfim, E. M., Freire, F. J., dos Santos, M. V. F., da Silva, T. J. A., \& dos Santos Freire, M. B. (2003). Avaliação de extratores para determinação de fósforo disponível de solos cultivados com Brachiaria brizantha. Acta Scientiarum. Agronomy, 25(2), 323-328.

Dechen, A. R., \& Nachtigal, G. R. (2007). Elementos requeridos à nutrição de plantas In: Novais, R. F., Alvarez, V. V. H., Barros, N. F., Fontes, R. L. F., Cantarutti, R. B., Neves, J. C. L. (Eds). Fertilidade do Solo. Viçosa: Sociedade Brasileira de Ciência do Solo - UFV. Cap. 03, p. 91-132.

Empresa Brasileira De Pesquisa Agropecuária Embrapa Soja. (2010). A importância do uso de semente de soja de alta qualidade. Boletim informativo folder n 01.

Farinelli R., Lemos L.B., Cavariani C., \& Nakagawa J. (2006). Produtividade e qualidade fisiológica de sementes de feijão em função de sistemas de manejo de solo e adubação nitrogenada. Revista brasileira de sementes, 28(2), 102-109.

Feltran, J. C., Maté u, G. P., Crusciol, C. A. C., Lemos, L. B., \& Silva, L. M. (2007). Root growth and micronutrient uptake by common bean as affected by liming. 35, 88-105.

Graciano, C., Goya, J. F., Frangi, J. L., \& Guiamet, J. J. (2006). Fertilization with phosphorus increases soil nitrogen absorption in young plants of Eucalyptus grandis. Forest Ecology and Management, 236(2-3), 202-210.

Grant, C. A., Flaté n, D. N., Tomasiewicz, D. J., \& Sheppard, S. C. (2001). A importância do fósforo no desenvolvimento inicial da planta. Better Crops with Plant Food.

Guoju, X., Fengju, Z., Juying, H., Chengke, L., Jing, W., Fei, M., \& Zhengji, Q. (2016). Response of bean cultures' water use efficiency against climate warming in semiarid regions of China. Agricultural water management, 173, 84-90.

Hammond, J. P., \& White, P. J. (2008). Sucrose transport in the phloem: integrating root responses to phosphorus starvation. Journal of experimental botany, 59(1), 93-109.

Henning, F. A., Mertz, L. M., Junior, E. A. J., Machado, R. D., Fiss, G., \& Zimmer, P. D. (2010). Composição química e mobilização de reservas em sementes de soja de alto e baixo vigor. Bragantia, 69, 727-734. 
Jaimez, R. E., Rada, F., García-Núñez, C., \& Azócar, A. (2005). Seasonal variations in leaf gas exchange of plantain cv. Hartón (Musa AAB) under different soil water conditions in a humid tropical region. Scientia Horticulturae, 104(1), 79-89.

Kikuti, H., Andrade, M. J. B., Carvalho, J. G., \& Morais, A. R. (2005). Nitrogênio e fósforo em feijão (Phaseolus vulgaris L.) variedade cultivada BRS MG Talismã. Acta Scientiarum Agronomy., 27, 415-422.

Kolchinski, E. M., Schuch, L. O. B., \& Peske, S., T. R. (2006). Early growth of soybean plants in relation to seeds vigor. Revista Bras. Agroc, $12,163-166$.

Krolow, R. H., Mistura, C., Coelho, R. W., Siewerdt, L., \& Zonta, E. P. (2004). Effect of phosphorus and potassium on development and nodulation of three cool season annual legumes. Revista Brasileira de Zootecnia, 33, 2224 - 2230.

Liao, H., Rubio, G., Yan, X., Cao, A., Brown, K. M., \& Lynch, J. P. (2001). Effect of phosphorus availability on basal root shallowness in common bean. Plant and Soil, 232, 69-79.

Liu, G. D., Morgan, K., Hogue, B., Li, Y. C., \& Sui, D. (2015). Improving phosphorus use efficiency for snap bean production by optimizing application rate. Hort. Sci. 42(2): 94-101.

Lynch, J., Lauchli, A., \& Epstein, E. (1991). Vegetative growth of the common bean in response to phosphorus nutrition. Crop Science. 31 , 380 - 387.

Machado E. C., Schmidt P.T., Medina C. L., \& Ribeiro R. V. (2005). Respostas da fotossíntese de três espécies de citros a fatores ambientais. Pesquisa Agropecuária Brasileira, Brasília, DF, 40(12), 1161- 1170.

Machado, V. J., de Souza, C. H. E., de Andrade, B. B., Lana, R. M. Q., \& Korndorfer, G. H. (2011). Curvas de disponibilidade de fósforo em solos com diferentes texturas após aplicação de doses crescentes de fosfato monoamônico. Bioscience Journal, 27(1).

Maluf, H. J. G. M., Soares, E. M. B., Silva, I. R., Neves, J. C. L., \& Silva, M. F. O. (2015). Disponibilidade e recuperação de nutrientes de resíduos culturais em solo com diferentes texturas. Revista Brasileira de Ciência do Solo, 39, 1690-1702.

Marcos Filho, Júlio. (1999). Testes de vigor: importância e utilização. Vigor de sementes: conceitos e testes.

Mei, P.-P., Gui, L.-G., Wang, P., Huang, J.-C., Long, H.-Y., Christie, P., \& Li, L. (2012). Maize/faba bean intercropping with rhizobia inoculation enhances productivity and recovery of fertilizer $\mathrm{P}$ in a reclaimed desert soil. Field Crops Research, 130, 19-27.

Mondo, V. H., Nascente, A., Neto, M. (2016). Common bean seed vigor affecting crop grain yield. Journal of Seed Science, 38(4), 365-370.

Neves, A. L., Lacerda, C. F. D., Guimarães, F. V., Gomes Filho, E., \& Feitosa, D. R. (2009). Trocas gasosas e teores de minerais no feijão-de-corda irrigado com água salina em diferentes estádios. Revista Brasileira de Engenharia Agrícola e Ambiental, 13, 873-881.

Novais, R. D., \& Smyth, T. J. (1999). Fósforo em solo e planta em condições tropicais (No. 631.422 N934). Universidade Federal de Vicosa, Vicosa, MG (Brasil). Departamento de Solos.

Oliveira, I. P., Edwards, D. G., Asher, C. J., Grundon, N. J., dos Santos, R. S. M., \& de Faria, C. D. (2001). Modos de aplicação e doses de fósforo no crescimento do feijoeiro. Embrapa Arroz e Feijão-Artigo em periódico indexado.

Pedó, T., Koch, F., Martinazzo, E. G., Villela, F. A., \& Aumonde, T. Z. (2015). Physiological attributes, growth and expression of vigor in soybean seeds under soil waté rlogging. African Journal of Agricultural Research, 10(39), 3791-3797.

Pommel, B., Mouraux, D., Cappellen, O., \& Ledent, J. F. (2002). Influence of delayed emergence and canopy skips on the growth and development of maize plants: a plant scale approach with CERES-Maize. European Journal of Agronomy, 16, 263-277.

Procópio, S., Santos, J., Pires, F., Da Silva, A., \& Mendonça, E. (2005). Absorção e utilização do fósforo pelas culturas da soja e do feijão e por plantas daninhas. Revista Brasileira de Ciencias do Solo, 29:911-921.

Ramos, S. J., Faquin, V., Rodrigues, C. R., Silva, C. A., Ávila, F. W. D., \& Sampaio, R. A. (2010). Utilização de fósforo e produção do feijoeiro: influência de gramíneas forrageiras e fontes de fósforo. Revista Brasileira de Ciência do Solo, 34(1), 89-96.

Razaq, M., Zhang, P., \& Shen, H. L. (2017). Influence of nitrogen and phosphorous on the growth and root morphology of Acer mono. PloS One, 12(2), 1-13.

Rezapour, R., Kazemi-Arbat, H., Yarnia, M., \& Zafarani-Moattar, P. (2013). Effect of seed size on germination and seed vigor of two soybean (glycin max L.) cultivars. Intl. Res. J. Appl. Basic. Sci. Vol., 4(11), 3396-3401.

Sant'Ana, E. V. P., dos Santos, A. B., \& da Silveira, P. M. (2010). Adubação nitrogenada na produtividade, leitura SPAD e teor de nitrogênio em folhas de feijoeiro. Embrapa Arroz e Feijão-Artigo em periódico indexado.

Santos, J. Z. L., Furtini Neto, A. E., Resende, Á. V. D., Carneiro, L. F., Curi, N., \& Moretti, B. D. S. (2011). Resposta do feijoeiro à adubação fosfatada em solos de cerrado com diferentes históricos de uso. Revista Brasileira de Ciência do Solo, 35, 193-202.

Scheeren, B. R., Peske, S., Schuch, L. O, \& Barros, A. C. (2010). Qualidade fisiológica e produtividade de sementes de soja. Revista Brasileira de Sementes, vol. 32 , n 3 p. 035-041.

Schlindwein, J. A., \& Gianello, C. (2009). Fósforo disponível determinado por lâmina de resina enterrada. Revista Brasileira de Ciência do Solo, 33 , 77-84.

Silva D, A., Esteves, J. A., Messias, U., Teixeira, A., Gonçalves, J., Chiorato, A. F., \& Carbonell, S. A. (2014). Efficiency in the use of phosphorus by common bean genotypes. Sci. Agric. 71(3), 232-239.

Silva, A. D., Brito, M. E. B., Frade, L. J. G., Nobre, R. G., Costa, F. B. D., Melo, A. S. D., \& Silva, L. D. A. (2016). Crescimento e trocas gasosas de genótipos de feijão-caupi sob estratégias de cultivo. Revista Ambiente \& Água, 11(3), 745-758. 
Research, Society and Development, v. 11, n. 2, e58011225914, 2022

(CC BY 4.0) | ISSN 2525-3409 | DOI: http://dx.doi.org/10.33448/rsd-v11i2.25914

Silva, D. A. D., Esteves, J. A. D. F., Messias, U., Teixeira, A., Gonçalves, J. G. R., Chiorato, A. F., \& Carbonell, S. A. M. (2014). Efficiency in the use of phosphorus by common bean genotypes. Scientia Agricola, 71(3), 232-239.

Silva, D. A., Tsai, S. M., Chiorato, A. F., da Silva Andrade, S. C., de Fatima Esteves, J. A., Recchia, G. H., \& Carbonell, S. A. M. (2019). Analysis of the common bean (Phaseolus vulgaris L.) transcriptome regarding efficiency of phosphorus use. PloS one, 14(1), e0210428.

Silva, D. A., Tsai, S. M., Chiorato, A. F., da Silva Andrade, S. C., de Fatima Esteves, J. A., Recchia, G. H., \& Carbonell, S. A. M. (2019). Analysis of the common bean (Phaseolus vulgaris L.) transcriptome regarding efficiency of phosphorus use. PloS one, 14(1).

Silva, E. L., de Araujo, A. F., dos Santos, V. B., Nunes, L. P. L., \& Carneiro, R. V. (2010). Biological N2 fixation in cowpea under different rates and sources of soluble phosphorus. Bioscience Journal, 26(3), 394-402.

Silva, P. R. C. (2006). Processo de produção de adubos foliares. Salvador: Serviço Brasileiro de Respostas Técnicas (Rede de Tecnologia da Bahia), 5 p.

Silva, R. (2002). Resposta do feijoeiro à adubação fosfatada num NEOSSOLO LITÓLICO distrófico da região sul do Rio Grande do Sul. Current Agricultural Science and Technology, 8(2), 129-132.

Silva, V. N., Zambiasi, C. A., Tillmann, M. A. A., Menezes, N. L., \& Villela, F. A. (2014). Condução do teste de condutividade elétrica utilizando partes de sementes de feijão. Revista de Ciências Agrárias, 37(2), 206-213.

Souza Ferraz, R. L., Melo, A. S., Suassuna, J. F., de Brito, M. E. B., Fernandes, P. D., \& Júnior, E. D. S. N. (2012). Trocas gasosas e eficiência fotossintética em ecótipos de feijoeiro cultivados no semiárido. Pesquisa Agropecuária Tropical, 42(2), 181-188.

Syers, J. K., Johnston, A. E., \& Curtin, D. (2008). Efficiency of soil and fertilizer phosphorus use. FAO Fertilizer and plant nutrition bulletin, 18(108).

Tunes, L. M., Barros, A. C. S. A., Badinelli, P. G. \& Olivo, F. (2008). Testes de vigor em função de diferentes épocas de colheita de sementes de cevada (Hordeum vulgare L.). Revista Brasileira de Ciências. Agrárias, 3, 321-326.

Urrutia, O., Guardado, I., Erro, J., Mandado, M., \& García-Mina, J. M. (2013). Theoretical chemical characterization of phosphate-metal-humic complexes and relationships with their effects on both phosphorus soil fixation and phosphorus availability for plants. Journal of the Science of Food and Agriculture, 93(2), 293-303.

Varennes, A., De Melo, J. P., \& Ferreira, M. (2002). Predicting the concentration and uptake of nitrogen, phosphorus and potassium by field-grown green beans under non-limiting conditions. European Journal of Agronomy. 17(1), 63-72.

Vian, A., Santi, A., Telmo Amado, J., Cherubin, M., Simon, D. H., Damian, H, \& Bredemeier, C. Variabilidade espacial da produtividade de milho irrigado e sua correlação com variáveis explicativas de planta. Ciência Rural, 46(3), 464-471.

Vitousek, P. M., \& Sanford, R. L. (1986). Nutrient cycling in moist Tropical forest. Annual Review of Ecology and Systematics, 17(2), 137-167

White, P. J., \& Hammond, J. P. (Eds.). (2008). The ecophysiology of plant - phosphorus interactions (vol. 7). Springer Science \& Business Media.

Zucareli, C., Prando, A. M., Júnior, E. U., \& Nakagawa, J. (2011). Phosphorus on the productivity and seed quality of bean Carioca Precoce cultivate d during the rainy season. Revista Ciência Agronômica, 42(1), 32. 